Proc. Indian Acad. Sci. (Chem. Sci.), Vol. 106, No. 5, October 1994, pp. 1203-1212.

(C) Printed in India.

\title{
Preparatively useful transformations of steroids and morphine alkaloids by Mucor piriformis
}

\author{
K M MADYASTHA \\ Department of Organic Chemistry, Indian Institute of Science, Bangalore 560012, India
}

\begin{abstract}
A versatile fungus isolated in our laboratory and identified as Mucor piriformis has been shown to effect novel and preparatively useful transformations in steroids and morphine alkaloids. The organism very effectively carries out hydroxylation of various $C_{19}$ and $\mathrm{C}_{21}$ steroids at 7 and 14-positions. Although the organism is capable of catalysing hydroxylation at $6 \beta$ and $11 \alpha$-positions, these are not the major activities. The $14 \alpha$-hydroxylase appears to have a broad substrate specificity. However, steroids with a bulky substitution at C-17 $\alpha$-position or without the 4-en-3-one group are not accepted as substrates by the $14 \alpha$-hydroxylase system. Studies have demonstrated how various $C_{19}$ and $C_{21}$ steroids can be modified to yield new structures which are either difficult to prepare by traditional methods or hitherto unknown. The organism also very efficiently and selectively carries out the $\mathrm{N}$-dealkylation of thebaine and its $\mathrm{N}$-variants. Interestingly, the nor-compound formed does not get further metabolized. Since thebaine is very often used as a starting material to synthesize various morphine agonists as well as antagonists, and one of the steps involved in their preparation is the $\mathrm{N}$-dealkylation reaction, the microbial process could certainly offer an alternative approach.
\end{abstract}

Keywords. Steroids; morphine alkaloids; Mucor piriformis; hydroxylation; N-dealkylation; transformation.

\section{Introduction}

In recent years, the most significant development that has taken place in the field of synthetic chemistry has been the application of biological systems to chemical reactions. One of the biological systems which appears to have the greatest potential in synthetic organic chemistry is microorganisms or enzymes isolated from them. Modification of natural products using microorganisms has been a very useful method in synthetic organic chemistry. Reactions catalyzed by microbes often offer significant advantages including those of efficiency, regiospecificity, stereoselectivity, etc. The conditions under which microbial reactions take place are mild and hence compounds sensitive to heat, acids and bases can be easily subjected to such transformations. Microbes and microbial enzymes are being used as reagents in various organic synthesis (Rosazza 1982; Yamada and Shimizu 1988; Crout and Christen 1989; Davies et al 1989). The scope of microbial reactions is widened considerably due to the finding that these reactions can also be carried out in organic solvents or in emulsions of water and immiscible organic solvents. Today, chemists are exploring the possibility of using microbes or enzymes isolated from them in the synthesis of different chiral synthons and physiologically as well as commercially important compounds.

The field of microbial transformation of steroids got a tremendous boost after the successful $11 \alpha$-hydroxylation of progesterone by the fungus, Rhizopus arrhizus 
(Peterson and Murray 1952). Transformation of steroids by various fungi have gained industrial importance since these methods can be used in the synthesis of steroidal hormones and their analogs. Hydroxylation at specific positions of a steroid molecule can be efficiently carried out using microorganisms. In fact, significant amount of work has already been carried out on the microbial steroid transformations and excellent reviews have appeared in the literature on this topic (Charney and Herzog 1967; Iizuka and Naito 1981; Mahato et al 1989).

In our efforts to find suitable microorganisms which can effect novel and useful transformations in some of the representative steroids, we isolated a fungal strain identified as Mucor piriformis which has been shown to effect novel and preparatively useful transformations of some of the steroids and morphine alkaloids. The present paper is confined to various transformations mediated by this versatile microorganism and the scope of its synthetic applicability. However, complete potential of Mucor piriformis as a tool in organic synthesis is yet to be established.

\section{Transformations of $\mathrm{C}_{21}$ and $\mathrm{C}_{19}$ steroids by Mucor piriformis}

Fungi belonging to the genus Mucor have been reported to mainly effect hydroxylation at $14 \alpha, 11 \alpha, 7 \alpha$ and $6 \beta$-positions of various steroids (Tamm et al 1963; Vezina and Singh 1975; Holland and Riemland 1985; Madyastha and Srivatsan 1987; Krishnan et al 1991). However, much information has been covered by patents (Murray and Peterson 1957; Dodson and Tweitt 1960; Charney and Herzog 1967). Mucor piriformis isolated in our laboratory has been shown to be versatile in effecting transformations in both $\mathrm{C}_{21}$ and $\mathrm{C}_{19}$ steroids. As far as we know only two reports have appeared in the literature on the transformations of steroids; viz. 21-hydroxy-4-pregnene-3,20dione (Murray and Peterson 1957) and 17 $\alpha$-21-dihydroxy-4-pregnene-3,20-dione (Eroshin 1962) by Mucor piriformis. These reports indicate the ability of this organism to introduce hydroxyl function at C-9, C-11 and C-6 positions. However, Mucor piriformis isolated in our laboratory is known to hydroxylate mostly at 7 and 14 positions in various $C_{19}$ and $C_{21}$ steroids.

\subsection{Transformations of progesterone (1)}

Mucor piriformis has been shown to transform progesterone (1) predominantly into $14 \alpha$-hydroxyprogesterone (2) which further gets hydroxylated at $6 \beta, 7 \alpha$ or $7 \beta$ positions, thereby yielding the corresponding dihydroxyprogesterones (figure 1) (Madyastha and Srivatsan 1987). The organism also produces $5 \beta, 14 \alpha$-dihydroxypregnane-3,20dione (6) as a minor metabolite. Time course experiments have clearly demonstrated that $14 \alpha$-hydroxylation is the first step involved in the transformation sequence (figure 1). In fact in $12 \mathrm{~h}$, nearly $75 \%$ of progesterone (1) gets metabolised mostly to $14 \alpha$-hydroxyprogesterone (2) whereas prolonging the incubation to $48 \mathrm{~h}$ results in the formation of dihydroxyprogesterones (figure 1 , compounds $\underline{3}, \underline{4}$ and $\underline{5}$ ). Transformation of progesterone ( $\underline{1}$ ) into compounds $\underline{5}$ and $\underline{6}$ (figure 1) by Mucorals has not been reported earlier. Earlier studies have indicated that $M$ ucor species have rigid stereoselectivity in their ability to hydroxylate various $C_{19}$ and $C_{21}$ steroids (Vezina and Singh 1975). However, it is interesting to note that $M$. piriformis isolated in our laboratory readily hydroxylates $14 \alpha$-hydroxyprogesterone (2) at both $7 \alpha$ and $7 \beta$-positions. 
<smiles>CC(=O)[C@H]1CC[C@]2(O)[C@H]3CCC4(O)CC(=O)CC[C@]4(C)C3CC[C@]12O</smiles>

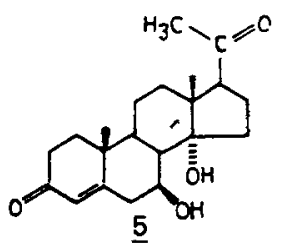

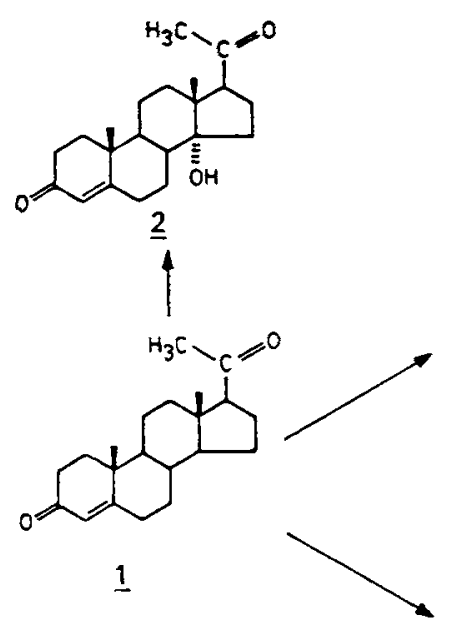<smiles>CC(=O)[C@H]1CC[C@]2(O)[C@H]3C[C@H](O)C4=C[C@@H](O)CC[C@]4(C)C3CC[C@]12C</smiles>

$\underline{3}$

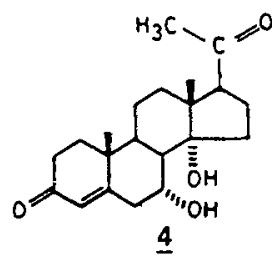

Figure 1. Transformations of progesterone (1) by $M$. piriformis.

\subsection{Transformations of $17 \alpha$-hydroxyprogesterone (}

Mucor piriformis has not been used to study the transformations in $17 \alpha$-hydroxyprogesterone (7), 16-dehydroprogesterone (12) and pregnenolone (17). Hence these studies have been carried out to establish whether or not a hydroxyl group at the $17 \alpha$-position or a C-16(17) double bond in a progesterone skeleton would effect the mode of transformation by this organism. It is also of interest to find out the functional significance of 4-en-3-one group in a $C_{21}$ steroid molecule and for this reason pregnenolone (17) which is devoid of this group has been chosen as the substrate.

The organism transforms (Madyastha and Joseph 1994) 17 $\alpha$-hydroxyprogesterone

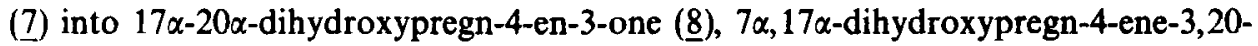

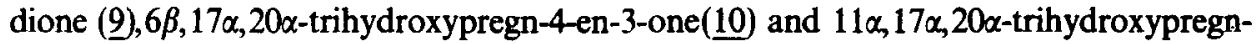
4-en-3-one (11) (figure 2). It is interesting to note that the presence of a hydroxyl at $17 \alpha$-position sterically hinders hydroxylation at $14 \alpha$-position. This is actually in accordance with the earlier report that an alkyl substitution at the $17 \alpha$-position in a steroid molecule prevents hydroxylation at the $14 \alpha$-position by $M$ ucor grisco-cyanus (Singh et al 1967). The proximity of the bulky $17 \alpha$-substituent probably hinders hydroxylation at $14 \alpha$-position and directs it to $7 \alpha$-position. However, steroids with hydroxyl group at $17 \beta$-position as in testosterone $(21)$ is readily transformed to its $14 \alpha$-hydroxy derivative (Krishnan et al 1991). Earlier, it has been demonstrated that incubation of $17 \alpha$-hydroxyprogesterone (7) with a Bacillus species results in the formation of a side-chain cleaved product (Mahato and Banerjee 1986). It appears that $M$. piriformis is not capable of cleaving the $C_{17}$ side-chain in a $C_{21}$ steroid. Surprisingly, the organism showed its unique ability to reduce the C-20 keto group, a reaction never been reported earlier in organisms of the order Mucorales. However, the reduction of C-20 keto group has not been observed in the case of progesterone (1), 16-dehydroxyprogesterone (12) and pregnenolone (17) (figures 1, 3 and 4). Time 


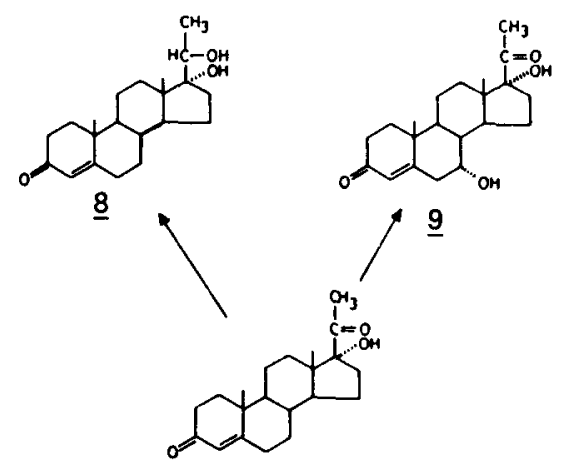

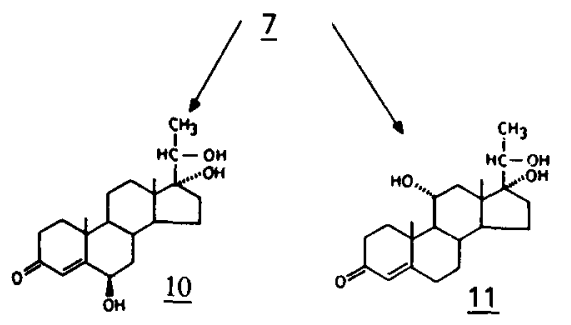

Figure 2. Transformations of $17 \alpha$-hydro xyprogesterone (7) by $M$. piriformis.
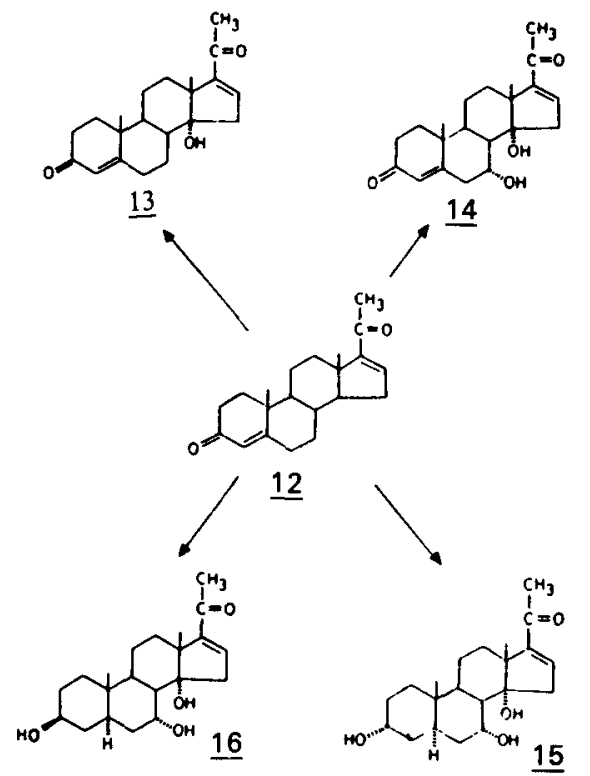

Figure 3. Transformations of 16-dehydropro gesterone (12) by $M$. piriformis.

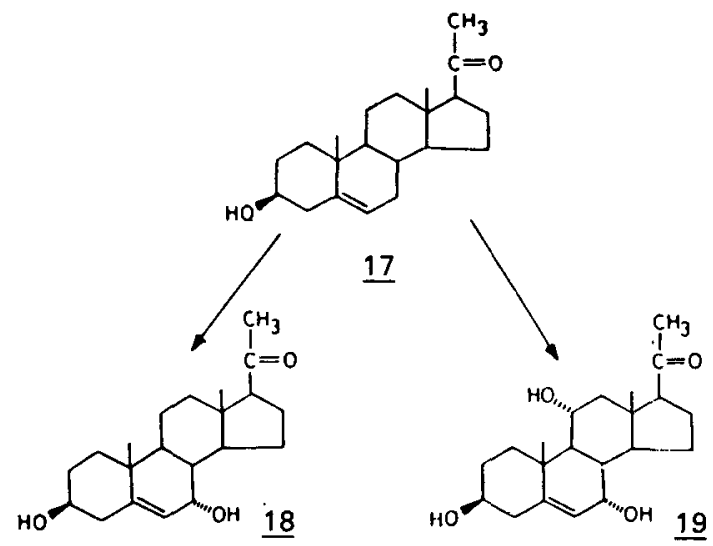

Figure 4. Transformations of pregnenolone (17) by $M$. piriformis.

course studies have indicated (Madyastha and Joseph 1994) that $17 \alpha, 20 \alpha$-dihydroxypregn-4-en-3-one ( $\underline{8}$, figure 2 ) is the initial biotransformation product formed which then gets hydroxylated either at the 6 $\beta$ - or $11 \alpha$-position (Madyastha and Joseph 1994).

\subsection{Transformations of 16-dehydroprogesterone (12)}

Various fungal species have been reported to transform 16-dehydroprogesterone (12) into different metabolites involving hydroxylation at $11 \alpha$ - and 21-positions, 
dehydrogenation at $\mathrm{C}-1$ position and reduction of the $\mathrm{C}-16(\underline{17})$ double bond (Kita and Shull 1959; Laskin 1963; Vezina et al 1963). In contrast to these observations, $M$. piriformis very efficiently carries out (Madyastha and Joseph 1994) 14 $\alpha$-hydroxylation of 16-dehydroprogesterone (12, figure 3). Time-course studies have indicated that the organism initiates the hydroxylation at the $14 \alpha$-position to yield $14 \alpha$-hydroxypregna4,16-diene-3,20-dione ( $\underline{13})$ which further gets hydroxylated at the $7 \alpha$-position (14, figure 3 ). In fact, at the end of $48 \mathrm{~h}$, nearly $80 \%$ of the substrate (12) gets transformed to $7 \alpha, 14 \alpha$-dihydroxypregna-4-16=diene-3,20-dione (14, figure 3$)$ and this metabolite gets accumulated in the fermentation medium in significant levels. The reduction of the 4-en-3-one group resulting in the formation of metabolites $\underline{15}$ and $\underline{16}$ (figure 3) is not a major activity of this organism since both these metabolites are formed in very low levels even at the end of $48 \mathrm{~h}$ (Madyastha and Joseph 1994). However, it is interesting to note that the reduction of the 3-keto group is not stereoselective since metabolites with both $3 \alpha$ - and $3 \beta$-hydroxyl functions have been isolated (figure 3 ). It is gratifying to, note that the metabolites $\underline{13}, \underline{14}, \underline{15}$ and $\underline{16}$, (figure 3 ), all derived from 16-dehydroprogesterone (12), appear to be hitherto unknown.

\subsection{Transformations of pregnenolone (17)}

To find out whether the organism has the ability to isomerize 5-en-3-ol to 4-en-3-one system in $\mathrm{C}_{19}$ and $\mathrm{C}_{21}$ steroids, representative steroids belonging to this category viz. pregnenolone (17) and dehydroepiandrosterone (26) have been used as substrates. These substrates have also provided an opportunity to find out the effect of a 5 -en-3 $\beta$-ol system on the mode of transformation by $M$. piriformis. Several reports have appeared in literature regarding the microbial conversion of pregnenolone (17) into progesterone (1) as a result of the isomerization of the $3 \beta$-hydroxy-5-ene to the 4-en-3-one system (Perlman 1952; Capek et al 1957). In addition to conversion of pregnenolone (17) to progesterone $(\underline{1})$, there are reports on the further conversion of progesterone $(\underline{1})$ to hydroxylated progesterones with hydroxyl function at $7 \beta, 11 \alpha, 12 \beta$, or $15 \alpha$-positions (Tan and Smith 1968; Namboori et al 1980).

The organism transforms pregnenolone (17) to $3 \beta, 7 \alpha$-dihydroxypregn-5-en-20-one $(\underline{18})$ and $3 \beta, 7 \alpha, 11 \alpha$-trihydroxypregn-5-en-20-one (19, figure 4) (Madyastha and Joseph, unpublished observation). $7 \alpha$-Hydroxylation seems to be one of the characteristic features of $M$. piriformis. Hydroxylation at the $7 \alpha$-position may not be due to the reactivity of the allylic position. The organism does not have the ability to cleave the $\mathrm{C}_{17}$ side-chain in pregnenolone (17, figure 4). Most of the earlier reports where pregnenolone (17) has been used as the substrate, progesterone $(\underline{1})$ has been shown to be formed as a result of the isomerization of the 5-en-3 $\beta$-ol system to the 4-en-3-one system (Perlman 1952; Tan and Smith 1968; Holland and Taylor 1979). M. piriformis appears to lack the isomerase and in this respect differs from the other organisms tested on various $\mathrm{C}_{21}$ steroids.

Cursory examination of the metabolites formed from different $\mathrm{C}_{21}$ steroids tested reveals that $M$. piriformis very efficiently carries out the $14 \alpha$-hydroxylation of $\mathrm{C}_{21}$ steroids. However, there appears to be a rigid structural requirement for the organism to accept substrates for $14 \alpha$-hydroxylation reaction. The organism readily converts steroids with a 4-en-3-one group and without bulky substitution at the $17 \alpha$-position to their respective $14 \alpha$-hydroxy derivatives. The organism is also known for its ability to carry out $7 \alpha$-hydroxylation. 


\subsection{Transformations of androstenedione (20)}

Androstenedione (20) appears to be a good substrate for M. piriformis. At a substrate concentration of $0.5 \mathrm{~g}$ per litre, virtually all the substrate added gets transformed into metabolites by the end of $24 \mathrm{~h}$. The organism transforms androstenedione (20) into testosterone (21), 14 $\alpha$-hydroxyandrostenedione (22), 7 $\alpha$-hydroxyandrostenedione (23), 14 $\alpha$-hydroxytestosterone $(24)$ and $7 \alpha, 14 \alpha, 17 \beta$-trihydroxyandrost-4-en-3-one (25) (figure 5) (Krishnan et al 1991; Madyastha and Joseph, unpublished observation). Time-course experiments carried out with androstenedione (20) have clearly indicated that during the early stages of incubation $(24 \mathrm{~h})$, hydroxylation takes place both at the $7 \alpha$-and $14 \alpha$-positions. However, the major metabolite formed at the end of $24 \mathrm{~h}$ has been shown to be $14 \alpha$-hydroxytestosterone ( 24 , figure $5,45 \%$ ). This metabolite (24) could have been formed from 22 (figure 5) in the presence of a 17-keto oxidoreductase or from testosterone (21) by $14 \alpha$-hydroxylation. The major metabolite formed at the end of $24 \mathrm{~h}$ viz. 14 $\alpha$-hydroxytestosterone (24) gets further hydroxylated at the $7 \alpha$-position resulting in the formation of a trihydroxy compound ( 25 , figure 5 , Madyastha and Joseph, unpublished observation). The level of this trihydroxy compound increases at the end of $48 \mathrm{~h}$ with concomitant decrease in the level of $\underline{24}$ (figure 5), suggesting a product precursor relationship.

The formation of testosterone (21) and 14 $\alpha$-hydroxytestosterone (24) from androstenedione (20) has been reported earlier using Phycomyces blakesleeanus (Smith et al 1989). Although the reduction of the 17-keto group has been reported in several

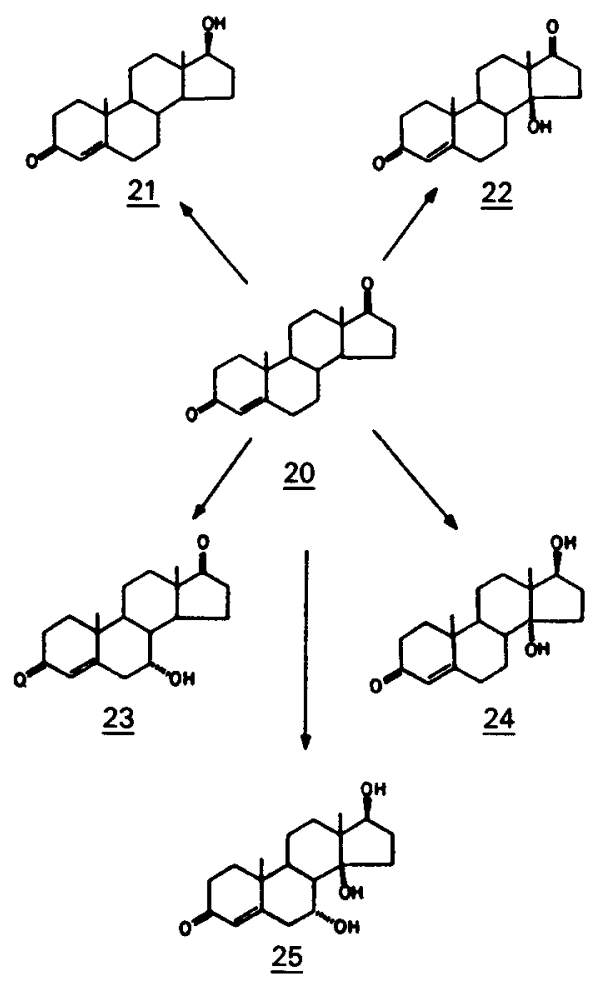

Figure 5. Transformations of androstenedione (20) by $M$. piriformis. 
microbial systems, it has never been shown to be present in $M$. piriformis. Similarly, the ability of microbes to carry out $14 \alpha$-hydroxylation of androstenedione (20) has been demonstrated earlier (Singh et al 1967; Crabb et al 1980). However, such an activity has never been shown in Mucor piriformis. The organism also accepts testosterone (21) readily as substrate and the mode of transformation is very similar to that observed with androstenedione $(\underline{20})$. The organism readily carries out hydroxylation at both $7 \alpha$ - and $14 \alpha$-positions. It is interesting to note that testosterone propionate is very poorly accepted as substrate (Madyastha and Joseph, unpublished observation).

\subsection{Transformations of dehydroepiandrosterone (26)}

Incubation of dehydroepiandrosterone (26) with $M$. piriformis yields mostly metabolites formed as a result of hydroxylation at the $7 \alpha$-position as well as reduction of the 17-keto group (figure 6) (Madyastha and Joseph, unpublished results). The organism transforms dehydroepiandrosterone $(\underline{26})$ into $3 \beta, 17 \beta$-dihydroxyandrost-5-ene $(\underline{27})$,

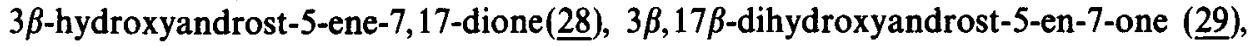
$3 \beta, 7 \alpha$-dihydroxy-androst-5-en-17-one $(\underline{30})$ and $3 \beta, 7 \alpha, 17 \beta$-trihydroxyandrost-5-ene (31). It is interesting to note that the organism fails to carry out $14 \alpha$-hydroxylation of dehydroepiandrosterone (26). Similar observation has also been made in the case of pregnenolone $(\underline{17})$. Both these compounds $(\underline{17}, \underline{26})$ contain 5 -en-3 $\beta$-ol system in their structure and it appears that one of the structural requirements needed for the $14 \alpha$-hydroxylase to accept a steroid molecule as a substrate is the presence of a

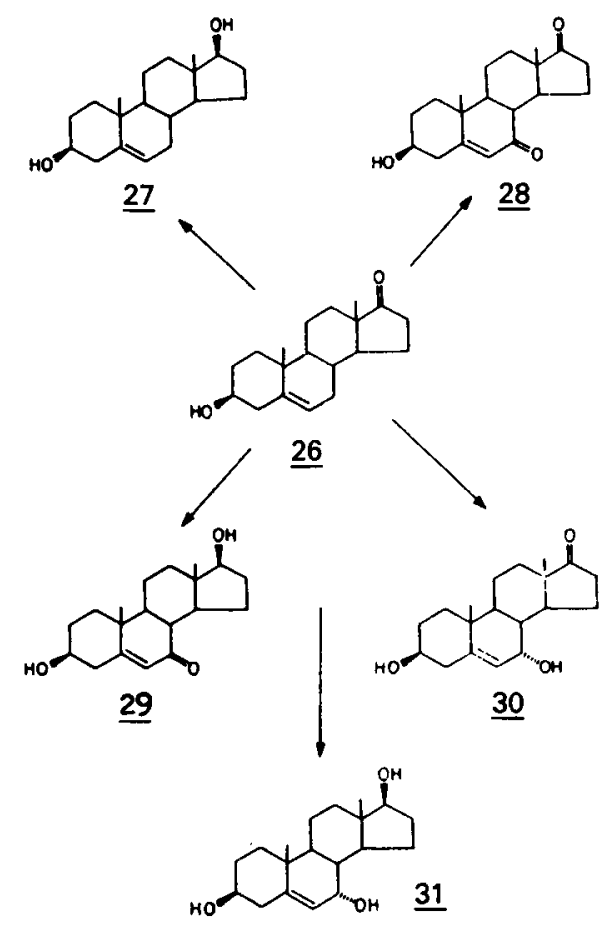

Figure 6. Transformations of dehydroepiandrosterone (26) by $M$. piriformis. 
4-en-3-one group in its molecule. This is supported by the fact that $M$. piriformis has the ability to carry out hydroxylation of progesterone (1), 16-dehydroprogesterone (12), androstenedione (20) and testosterone (21) at $14 \alpha$-position. The other interesting aspect is that the organism is not capable of isomerizing 5-en-3 $\beta$-ol to 4-en-3-one, a reaction many organisms are capable of performing. In the case of dehydroepiandrosterone (26) which is devoid of 4-en-3-one group, the organism prefers to carry out hydroxylation at $7 \alpha$-position. This $7 \alpha$-hydroxylation may not be due to the reactivity of the allylic position, but rather due to the geometrical nature of the active site. Earlier it has been shown that a Rhizopus species transforms dehydroepiandrosterone

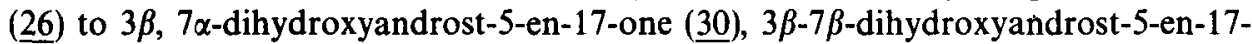
one and $3 \beta$-hydroxyandrost-5-ene-7,17-dione (28) (Dodson et al 1959). Thus 7hydroxylation of dehydroepiandrosterone (26) appears to be stereospecific. Absidia regnieri, belonging to the order Mucorales has been shown to transform dehydroepiandrosterone (26) into its $7 \alpha$-hydroxylated derivative (Bell et al 1975). The reduction of the 17-keto group of dehydroepiandrosterone (26) to $17 \beta$-hydroxyl has not been reported by fungi of the order Mucorales.

Studies carried out with $M$. piriformis demonstrated the versatility of this organism in introducing hydroxyl groups at the $7 \alpha$ - and $14 \alpha$-positions in various $C_{19}$ and $C_{21}$ steroids. Transformation of $17 \alpha$-hydroxyprogesterone (7) and 16-dehydroprogesterone (12) by this organism has resulted in the formation of metabolites $(\underline{11}, \underline{13}, \underline{14}, \underline{15}$ and 16) which are hitherto unknown. In fact, $M$. piriformis can be used as an efficient reagent to prepare some of these novel compounds.

\section{14a-Hydroxylation by cell-free extract of Mucor piriformis}

Mucor piriformis isolated in our laboratory has the unique ability to hydroxylate various $C_{19}$ and $C_{21}$ steroids (Madyastha and Srivatsan 1987; Krishnan et al 1991; Madyastha and Joseph 1994). Cell-free extract prepared from induced vegetative cell cultures of $M$. piriformis following the procedure developed earlier (Jayanthi et al 1982; Madyastha et al 1984; Madyastha and Joseph 1993) has been shown to contain

Table 1. Substrate specificity of the microsomal $14 \alpha$-hydroxylase from Mucor piriformis.

\begin{tabular}{lc}
\hline Substrate & $\begin{array}{c}14 \alpha \text {-hydroxylated product formed } \\
\text { (\% conversion) }\end{array}$ \\
\hline Progesterone & 69 \\
Testosterone & 65 \\
Androstenedione & 30 \\
16-Dehydroprogesterone & 81 \\
1,2-Dehydrotestosterone & 45 \\
17 $\alpha$-Hydroxyprogesterone & - \\
Epitestosterone & - \\
17 $\alpha$-Ethynyl-19-nortestosterone & - \\
$3 \beta$-Hydroxyandrost-5-ene-7,17-dione & - \\
\hline
\end{tabular}

The above experiments were carried out using acetone-washed microsomes as described earlier (Madyastha and Joseph 1993). 
high $14 \alpha$-hydroxylase activity. Most of the $14 \alpha$-hydroxylase activity is associated with the microsomes $(105,000 \mathrm{~g}$ sediment) prepared from the active cell-free extract. Both NADPH and $\mathrm{O}_{2}$ are necessary for the hydroxylase activity (Madyastha and Joseph 1993). Microsomes readily convert various $C_{19}$ and $C_{21}$ steroids to their respective $14 \alpha$-hydroxy compounds (table 1) (Madyastha and Joseph 1993). Microsomes prepared from the uninduced cells are devoid of $14 \alpha$-hydroxylase activity.

\section{Transformation of thebaine and its $\mathbf{N}$-variants}

The work presented so far clearly demonstrates the unique ability of $M$. piriformis to carry out preparatively useful steroid transformations. To our great surprise we have now demonstrated that this organism can be used as an efficient reagent for effecting N-dealkylation of thebaine ( 22 ), an isoquinoline alkaloid, and its N-variants where the alkyl group on nitrogen is varied from methyl to ethyl, $n$-propyl, isopropyl, $n$-butyl and cyclopropylmethyl (figure 7a). The organism essentially carries out $\mathrm{N}$ dealkylation resulting in the formation of norcompound (33) with high yields $(\sim 80)$ (Madyastha and Reddy 1994). It is interesting to note that the size of the alkyl group on nitrogen does not have any significant effect on the $\mathrm{N}$-dealkylation reaction. The most significant part of this microbial system is that the northebaine ( $\underline{33})$ formed does not get further metabolized. The organism also carries out $\mathrm{N}$-demethylation of the Diels-Alder adduct of thebaine (32) in high yields (figure 7b) (Madyastha and Reddy 1994).

Thebaine (32) is extensively used as a starting material to synthesize various morphine agonists and antagonists. One of the steps involved in their preparation

(a)

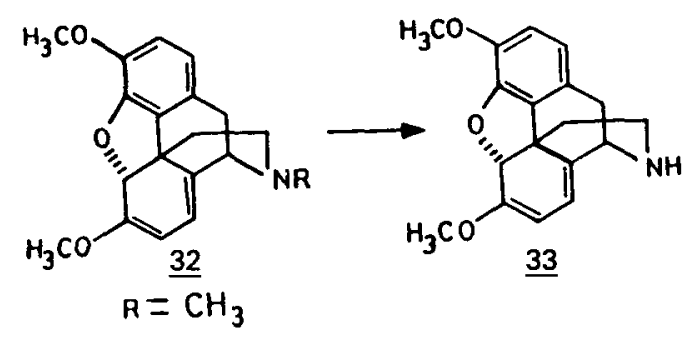

\begin{tabular}{ll}
\hline \multicolumn{1}{c}{$R$} & Yield $(\%)$ \\
\hline $\mathrm{CH}_{3}$ & 81 \\
$\mathrm{CH}_{2} \mathrm{CH}_{3}$ & 83 \\
$\mathrm{CH}_{2} \mathrm{CH}_{2} \mathrm{CH}_{3}$ & 86 \\
$\mathrm{CH}\left(\mathrm{CH}_{3}\right)_{2}$ & 88 \\
$\mathrm{CH}_{2} \mathrm{CH}_{2} \mathrm{CH}_{2} \mathrm{CH}_{3}$ & 89 \\
$\mathrm{CH}_{2}-$ & 92 \\
\hline
\end{tabular}

(b)

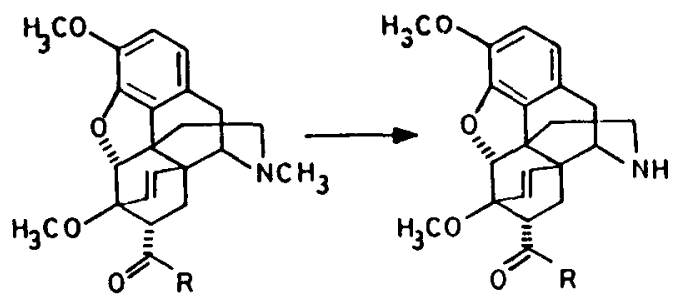

\begin{tabular}{ll}
$\mathrm{R}$ & Yield (\%) \\
\hline $\mathrm{CH}_{3}$ & 83 \\
$\mathrm{OCH}_{3}$ & 62
\end{tabular}

Figure 7. (a) $\mathrm{N}$-dealkylation of thebaine (32) and its $\mathrm{N}$-variants by $M$. piriformis. (b) $\mathrm{N}$-demethylation of the Diels-Alder adduct by $M$. piriformis. 
is the $\mathrm{N}$-dealkylation reaction which is normally carried out chemically. Since the chemical method involves harsh reaction conditions as well as hazardous and toxic reagents, microbial method could offer alternative approach. Our studies have demonstrated the suitability of $M$. piriformis as an efficient reagent to carry out $\mathrm{N}$-dealkylation of thebaine $(\underline{32})$ and its $\mathrm{N}$-variants.

\section{References}

Bell A M, Jones Sir E R H, Meakin G D, Miners J O and Wilkins A L 1975 J. Chem. Soc., Perkin I 2040

Capek A, Hanc O and Pavlu H 1957 Cesk. Microbiol. 2168

Charney W and Herzog H L 1967 In Microbiol. transformations of steroids: A handbook (New York: Academic Press)

Crabb T A, Dawson P J and Williams R O 1980 J. Chem. Soc. Perkin I 2535

Crout D H G and Christen M 1989 In Biotransformations in organic synthesis (ed.) R. Schefold (Berlin: Springer) pp. 1-114

Davies H G, Green R H, Kelly D R and Roberts S M 1989 In Biotransformations in preparative organic chemistry. The use of isolated enzymes and whole cell systems in synthesis (London: Academic Press) p. 99

Dodson R M, Nicholson R T and Muir R D 1959 J. Am. Chem. Soc. 816295

Dodson R M and Tweitt R C 1960 US Patent 2,924,611; 1960 Chem. Abstr. $5411086 \mathrm{~h}$

Eroshin V K 1962 Mikrobiologiya 31608

Holiand H L and Riemland E 1985 Can. J. Chem. 631121

Holland H L and Taylor G J 1979 Phytochemistry 18437

Iizuka H and Naito A 1981 In Microbial Conversion of Steroids and Alkaloids (University of Tokyo Press)

Jayanthi C R, Madyastha P and Madyastha K M 1982 Biochem. Biophys. Res. Commun. 1061262

Kita D A and Shull G M 1960 US Patent 2,903,398 (Sept. 8, 1959); 1960 Chem. Abstr. 54P 3520a

Krishnan R, Madyastha K M, Seshadri T P and Viswamitra M A 1991 Steroids 56440

Laskin A I 1962 US Patent 3,056,730 (Oct. 2, 1962); 1963 Chem. Abstr. 58P 8388f

Madyastha K M, Jayanthi C R, Madyastha P and Sumathi D 1984 Can. J. Biochem. Cell Biol. 62100

Madyastha K M and Joseph T 1963 J. Steroid Biochem. Mol. Biol. 45563

Madyastha K M and Joseph T 1994 Appl. Microbiol. Biotechnol. (in press)

Madyastha K M and Reddy G V B 1994 J. Chem. Soc., Perkins I (in press)

Madyastha K M and Srivatsan J 1987 Can. J. Microbiol. 33361

Mahato S B and Banerjee S 1986 Biochem. J. 239473

Mahato S B, Banerjee S and Poddar S 1989 Phytochemistry 287

Murray H C and Peterson D H 1952 US Patent 2602,769; 1952 Chem. Abstr. $468331 \mathrm{f}$

Murray H C and Peterson D H 1957 US Patent 2,800,490

Namboori K, Pereira L and Merchant J R 1980 Indian J. Biochem. Biophys. 17149

Perlman D 1952 Science 115529

Peterson D H and Murray H C 1952 J. Am. Chem. Soc. 741871

Rosazza J P 1982 In Microbial transformations of bioactive compounds (Boca Raton, Fl: CRC Press) vol. 1 and 2

Singh K E, Sehgal S N, Vezina C 1967 Can. J. Microbiol. 131271

Smith K E, Latif S and Kirk D N 1989 J. Steroid Biochem. 32445

Tamm C, Gubler A, Juhasz G, Weiss-Berg E and Zurcher W 1963 Helv. Chim. Acta. 46889

Tan L and Smith L L 1968 Biochem. Biophys. Acta. 164389

Vezina C, Sehgal S N and Singh K 1963 Appl. Microbiol. 1150

Vezina C and Singh K 1975 In The filamentous fungi (eds) J E Smith and D R Berry (London: Edward Arnold) vol. 1, p. 158

Yamada H and Shimizu S 1988 Angew. Chem., Int. Ed. Engl. 27622 